\title{
SISTEMA INSTITUCIONAL DE EVALUACIÓN DE COMPETENCIAS SIEC
}

\author{
JOHANN LEONARDO CAMPOS ALVARADO
}

\begin{abstract}
The Cooperative University of Colombia designs, builds and implements an evaluation system that ensures an epistemic, methodological and technical foundation for the internal evaluation of courses and at the institutional level. The main objective is to provide valid and reliable information on learning outcomes, which allows making decisions aimed at constantly improving the quality of education. The system builds and applies assessment instruments based on national and international quality standards, to evaluate generic, transversal and specific competences that are developed in the different academic programs. Additionally, it promotes this exercise as a permanent training action, promoting learning and moving away from the concept of control and qualification
\end{abstract}

Keywords:

Competences; evaluation; instruments; system; model

\section{RESUMEN}

La Universidad Cooperativa de Colombia, diseña y construye un sistema de evaluación que, de acuerdo con su Modelo Educativo Crítico con Enfoque de Competencias, asegura un fundamento epistémico, metodológico y técnico para desplegar la evaluación formativa al interior de los cursos (evaluación interna) y a nivel institucional (evaluación externa). El sistema construye y aplica instrumentos de evaluación con estándares nacionales e internacionales de calidad, validez teórica y empírica, procesamiento y divulgación, para evaluar las competencias genéricas, transversales y específicas que se desarrollan en los diferentes cursos. El sistema dirige su acción a profesores y estudiantes, es un elemento dentro del sistema de evaluación integral que la universidad diseñó para obtener una evaluación de 360 grados que permita valorar la acción de la universidad frente a su compromiso con el aprendizaje en los estudiantes.

Palabras clave:

Competencias; evaluación; instrumentos; sistema; modelo

\footnotetext{
${ }^{1}$ Magister, Universidad Cooperativa de Colombia, johann.camposa@ucc.edu.co , Colombia
} 
La Universidad Cooperativa de Colombia trazó su camino hacia la excelencia académica, con el propósito de responder a las expectativas de los estudiantes frente a su proceso de aprendizaje en la educación superior, y al llamado de la sociedad que demanda personas cada vez más competentes. En este sentido, la institución desarrolla el Sistema Institucional de Evaluación de Competencias - SIEC, convencida que la evaluación, especialmente en su dimensión formativa, es el mejor monitor para identificar la trascendencia del proyecto educativo de la universidad, observable en el desarrollo de las competencias de los estudiantes. En su construcción, la universidad se asegura que el sistema se encuentre en consonancia con los estándares nacionales e internacionales de calidad en sistemas de evaluación, en búsqueda de trascender los métodos tradicionales de calificación hacia una evaluación objetiva, a partir de procesos científicos e intersubjetivos.

Así pues, la universidad construye el SIEC y lo estructura en torno a los diferentes procesos y momentos en que la evaluación se considera un eje central; de esta manera se establecen cuatro áreas de trabajo, a saber: 1. Pruebas de competencias (diseño de instrumentos de evaluación); 2. Reconocimiento de competencias previas; 3. Rúbrica institucional y 4. Valor agregado.

La estructura del sistema se planea desde la concepción del Modelo Educativo Crítico con Enfoque de Competencias de la universidad, y comienza su preparación con la construcción de la base teórica en evaluación y construcción de instrumentos válidos y confiables, para lo cual se establece una estructura funcional: un asesor externo experto, Dr. Daniel Bogoya, un jefe nacional del SIEC, decanos nacionales y grupo de profesores expertos por áreas en todo el país. El SIEC se encarga del diseño y aplicación de instrumentos de evaluación (bloques de contextos e ítems) de competencias genéricas y transversales; así mismo acompaña el diseño de las rúbricas de los cursos bajo las cuales se despliega la evaluación interna. Dicho material se encuentra soportado en marcos teóricos, uno por cada instrumento. El procesamiento de los resultados se hace a partir de la Teoría de Respuesta al Ítem (TRI), y una vez aplicadas las pruebas, se hace el análisis de validez empírica de los instrumentos (ítems y constructos), se determinación las escalas y se asignan las calificaciones; finalmente se elaboran los reportes y se comunican a la comunidad académica. 
${ }^{2}$ La implementación de un sistema para evaluar el nivel de desarrollo de competencias, con estándares internacionales para el diseño, validación y calibración de ítems, verificación de validez de constructo, generación de escalas y elaboración de reportes en línea para los distintos destinatarios, constituye una herramienta de gestión para el cumplimiento de la misión de la Universidad, en términos de su responsabilidad social, para atender requerimientos de instancias gubernamentales sobre el cumplimiento de estándares mínimos y para lograr el reconocimiento de alta calidad, frente a la Comisión Nacional Intersectorial de Aseguramiento de la Calidad de la Educación Superior y el Consejo Nacional de Acreditación (CNA, 2006). En la perspectiva de acreditación internacional, también será clave contar con un sistema robusto de evaluación en operación, cuyos resultados se utilizan para evidenciar fortalezas y debilidades, constatar la efectividad de las estrategias desplegadas y acometer los ajustes respectivos.

La evaluación basada en instrumentos estandarizados y confiables lleva a que los profesores puedan dedicar su capacidad, tiempo y esfuerzo a la educación de los estudiantes, utilizando los resultados para mejorar las prácticas de aula y lograr un valor académico agregado importante, confiando la evaluación a una dependencia y un grupo de expertos especializados en el campo, que sean responsables de administrar el Sistema en forma técnica, oportuna y eficiente. De este modo, será posible diseñar nuevas estrategias de trabajo en aula, con base en el conocimiento de resultados parciales y acumulados de competencias desarrolladas, así como de las áreas y campos del saber débiles, en especial para casos críticos que no han respondido a las estrategias empleadas. Desde luego, los profesores pueden realizar exámenes adicionales en las aulas, para evaluar aspectos que se consideren necesarios y que escapan al alcance del Sistema; en este caso, la calificación del estudiante puede obtenerse combinando dos componentes: primero, el resultado entregado por el Sistema; y segundo, los resultados de los exámenes adicionales mencionados.

Como consecuencia del enfoque, se retoma la discusión acerca del papel de la evaluación: ¿debe ser formativo, como corresponde al desarrollo de competencias?; o ¿debe ser sumativo, propio de los contenidos? En la perspectiva de un papel formativo, un estudiante que

\footnotetext{
${ }^{2}$ (Bogoya, 2019, p. 7). Arquitectura Conceptual| Universidad Cooperativa de Colombia.
} 

en su esfuerzo y dedicación, visible con resultados crecientes en los distintos exámenes que presenta durante un semestre, ipuede ser certificado con nota aprobatoria! Al respecto, es necesario mencionar la fidelidad del sentido del Sistema con una visión formativa, respecto de las funciones de la evaluación establecidas en el Proyecto Institucional, PI, cuando se señala:

Medir y comparar los logros obtenidos. La evaluación establece el grado de logro de las competencias formuladas por el diseñador del curso. Se trata de observar hasta dónde ha alcanzado el estudiante el horizonte de sentido propuesto. La evaluación establece la situación del evaluado dentro de una escala de valores previamente existente. En el caso de las competencias, lo que se busca es mirar el grado de refinamiento de las mismas. (UCC, 2013, p. $30)$.

Para cumplir con este enunciado se espera la conjunción coordinada de esfuerzos de los distintos programas académicos, que conduzcan a evaluar las competencias de los estudiantes, mediante criterios institucionales y técnicas robustas, con instrumentos que satisfacen estándares internacionales de validez, al nivel de los ítems individuales y de los constructos definidos. También se requiere implementar protocolos de aplicación y métodos de validación, generación de escalas y asignación de notas que permitan entregar resultados confiables y oportunos, para retroalimentar el estado del proceso educativo a los estudiantes y conocer la efectividad de las estrategias de aula empleadas por los profesores, así como poder llevar registros y realizar estudios de tendencias con escalas equiparables (OCDE, 2017; Bogoya, 2008).

\section{OBJETIVOS}

\subsection{General.}

Como objetivo general se prevé brindar a la comunidad académica información verificable, válida y confiable sobre los resultados de aprendizaje, para permitir a la universidad tomar decisiones orientadas a mejorar la calidad de la educación de manera constante.

\subsection{Específicos.}

- Diseñar, construir y actualizar permanentemente instrumentos de evaluación que cuenten con validez teórica y técnica, para evaluar competencias genéricas y transversales de acuerdo con el mapa de competencias de los programas de la universidad.

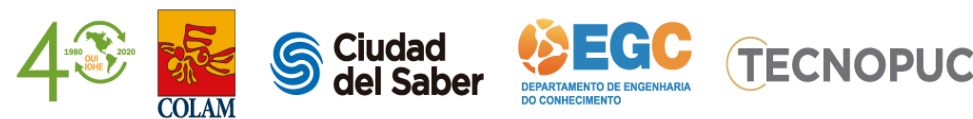


- Identificar la variabilidad de los resultados de aprendizaje a lo largo de la vida del estudiante en la universidad, para aportar en la medición del valor agregado en el proceso de formación; de esta manera reconocer el progreso, retroceso o estancamiento del desempeño de los estudiantes (variabilidad / consistencia), también como indicador de la calidad y eficacia de la acción educativa desplegada por la universidad.

- Usar el sistema de evaluación para reconocer las competencias previas de los aspirantes a los programas de la universidad, a través de la aplicación de los instrumentos dentro de protocolos establecidos de manera colegiada con un comité de validación, conformado por expertos de las áreas y personal del sistema institucional de evaluación de competencias.

- Acompañar y asesorar a los programas en el diseño de las rúbricas de cada uno de los cursos, las cuales sustentan la evaluación interna.

\section{DESCRIPCIÓN DE LA EXPERIENCIA}

En relación con el desarrollo de competencias de los estudiantes, entre el inicio y el término de un ciclo específico, la evaluación es el monitor de seguimiento para determinar la trascendencia del proyecto educativo y poder gobernar acciones de mejoramiento. En la perspectiva referida, la Universidad implementó el SIEC en el año 2016. En el primer semestre, conformados los equipos respectivos de profesores, se elaboró el marco teórico para los instrumentos que evalúan competencias genéricas de lectura crítica y razonamiento cuantitativo, se definieron tablas de especificaciones con una propuesta de distribución de ítems por campo conceptual (Bourdieu, 2002; Vergnaud, 1990) y dominios cognitivos taxonomía SOLO (Biggs, 2005; Hattie \& Brown, 2004), se seleccionaron contextos apropiados para las áreas consideradas (van Dijk, 2001; Wilson \& Sperberg, 2004) y se construyeron 160 ítems articulados en ocho bloques de 20 ítems cada uno, cuatro bloques para el instrumento de lectura y cuatro para el de razonamiento.

En el segundo semestre se afinó el marco teórico y se avanzó en la construcción de cuatro nuevos bloques, dos para lectura y dos para razonamiento. También en el segundo semestre, se conformaron equipos de profesores para elaborar el instrumento de evaluación de competencia genérica de comunicación escrita y dos instrumentos de competencias transversales de ingeniería: modelado matemático, simulación y optimización; y formulación y evaluación de proyectos. El Sistema entró en operación en el segundo semestre del mismo año

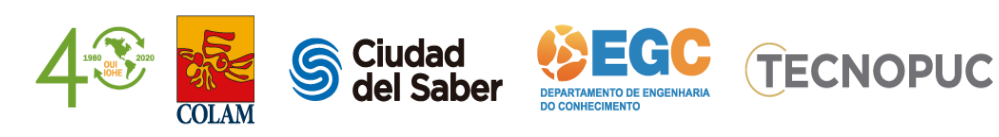


2016, con la evaluación de tres poblaciones: estudiantes que presentarían el examen Saber Pro en noviembre; estudiantes que ingresaron a primer semestre; y profesores de distintas sedes y programas. Los profesores fueron evaluados en el mes de noviembre, por una parte, y los estudiantes tanto en agosto como en noviembre, por la otra. Durante el año 2017, el Consejo Superior genera la norma interna del Sistema Institucional de Evaluación de Competencia y en desarrollo del acuerdo superior, se mantuvieron los equipos de profesores que venían trabajando y se conformaron ocho nuevos grupos para elaborar marcos teóricos, tablas de especificaciones y los respectivos instrumentos de evaluación de dos competencias genéricas y seis competencias transversales. Durante el año 2018 se mantuvieron algunos equipos, se reestructuraron otros y se conformaron dos nuevos grupos para realizar las tareas propias de los instrumentos de competencias transversales para el programa Crossmedia. (Bogoya, 2019.)

La estructura de diseño de instrumentos a partir de colegiaturas de expertos, lideradas por la jefatura del sistema y por el asesor experto externo, sigue siendo la estructura de acción permanente en construcción de instrumentos, en seguimiento y valoración de la experiencia. En 2019 se construyeron ocho instrumentos para competencias transversales, dos para el área de Ciencias Económicas Administrativas y Contables, dos para programas de salud, dos para ingenierías y dos para Ciencias Sociales. Paralelo a la construcción de instrumentos, desde el año 2016 se han venido aplicando a toda la población estudiantil de la Universidad, dos pruebas en 2016, una en 2017, cuatro en 2018 y dos en 2019, pruebas que han tenido 47.104 aplicaciones por parte de los estudiantes. A partir del 2019 se definió una estrategia para la construcción de las rúbricas para las competencias específicas, las cuales se aplican al interior de los cursos, e inician su proceso de validación teórica para recoger resultados de las prácticas durante el segundo semestre de 2020 y el primer semestre de 2021 y así iniciar la validación empírica o estadística de dichos instrumentos. Dentro del ejercicio de reconocimiento de competencias previas, el SIEC acompañó un caso aplicado de validación de estudios a un grupo de egresados de diseño del SENA, aspirantes al programa de Diseño Crossmedia de la Universidad. Con expertos del programa se construyó un protocolo de validación, para analizar el mapa de competencias que los aspirantes traían de su formación anterior, utilizando instrumentos del SIEC para validar las competencias que se definieron como validables según los resultados del estudio, en este caso, las competencias genéricas desplegadas en los cursos de humanidades.

En relación con el valor agregado, se han calculado y publicado informes según los resultados obtenidos, especialmente en 2019 se trabajó con un concepto diferente al de valor agregado como se conoce, más como un concepto de variabilidad en los resultados, no solo de 


\section{ciki}

X Congreso internacional de conocimiento e innovación
Ciudad del Saber, Panamá 19 y 20 de noviembre 2020

entrada y salida según las pruebas externas de Saber 11 y PRO, sino de las diferencias de cada uno de los resultados durante todo el proceso, esto ha generado mayor aceptación por parte de los estudiantes, ya que son ellos mismos los que proyectan la meta para mejorar sus resultados año tras año durante toda su estancia en la Universidad.

Tabla 1. Acciones para el reconocimiento de competencias

\begin{tabular}{|c|l|}
\hline Acciones & \multicolumn{1}{|c|}{ Descripción } \\
\hline $\begin{array}{c}\text { Examen de clasificación, validación y } \\
\text { suficiencia mediante pruebas SIEC e } \\
\text { ITEP internacional para los cursos de } \\
\text { inglés }\end{array}$ & Acuerdo institucional 459 de 2019 \\
\hline $\begin{array}{c}\text { Reconocimiento de competencias } \\
\text { previas en reglamento estudiantil }\end{array}$ & $\begin{array}{l}\text { Establecer condiciones de reconocimiento de } \\
\text { competencias vía pruebas SIEC (experiencias y } \\
\text { estudios previos), certificaciones y convenios }\end{array}$ \\
\hline Calibración pruebas SIEC-Saber 11 & Análisis de correspondencia entre pruebas \\
\hline $\begin{array}{c}\text { Pruebas de reconocimiento de } \\
\text { competencias en Gestión Financiera e } \\
\text { Ingenierías }\end{array}$ & $\begin{array}{l}\text { Unidad de reconocimiento de competencias } \\
\text { previas: Decanos nacionales, miembros SIEC y } \\
\text { profesores (jurados y correctores) } \\
\text { Protocolo: 4 bloques, prueba de escritura con } \\
\text { rúbrica y sustentación oral con rúbrica }\end{array}$ \\
\hline $\begin{array}{c}\text { Definición de criterios y } \\
\text { procedimientos para la validación de } \\
\text { competencias de los aspirantes del } \\
\text { SENA al programa de Crossmedia en } \\
\text { campus Medellín }\end{array}$ & $\begin{array}{l}\text { Apoyo al documento presentado por la } \\
\text { coordinación del programa Crossmedia y la } \\
\text { Subdirección académica del campus Medellín, } \\
\text { para generar el acuerdo de reconocimiento de } \\
\text { competencias previas en acuerdo institucional } \\
\text { (protocolo de validación para Crossmedia 2019) }\end{array}$ \\
\hline
\end{tabular}

Fuente: Universidad Cooperativa de Colombia. Informe condiciones iniciales. Anexo 20 SIEC

El propósito principal de la acción del SIEC, además de diseñar, construir, aplicar instrumentos y generar reportes, es el de generar análisis permanentes de los resultados al interior de los diferentes cuerpos colegiados como comités de área, comités curriculares, consejos académicos, etc. La dinámica del SIEC se muestra en la siguiente gráfica, la cual resume la actividad desde el 2016. 
Figura 1. Sistema Institucional de Evaluación de Competencias SIEC

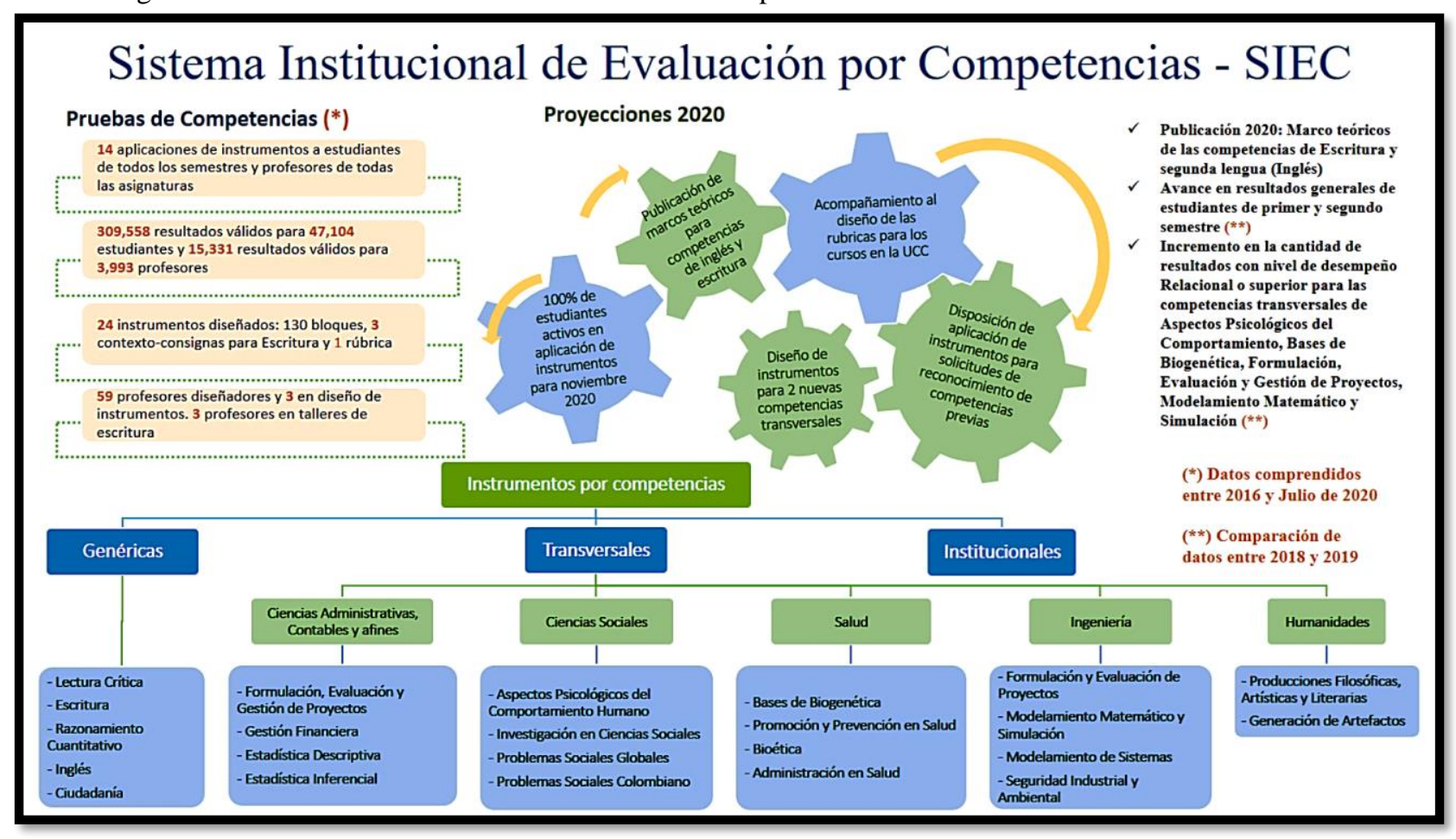

Fuente: Universidad Cooperativa de Colombia. Informe condiciones iniciales. Anexo 20 SIEC

\section{RESULTADOS}

A la fecha la Universidad ha transformado el discurso de los profesores y estudiantes frente a la evaluación, toda vez que la comunidad en general ahora comprende y promueve la aplicación de los instrumentos de manera periódica, para conocer los resultados del estado del proceso en cada uno de los estudiantes, también se reconoce el impacto real de la acción institucional.

Se han diseñado y aplicado dieciocho instrumentos que evalúan las competencias transversales, cinco que valoran las genéricas y uno que valora las competencias institucionales. Los resultados obtenidos han mostrado el estado del desarrollo de dichas competencias durante cinco años, y han sido de vital importancia para reorientar y afinar los planes de curso, así como para diseñar nuevas estrategias institucionales que mejoren los procesos más sensibles.

Actualmente se adelanta el acompañamiento y asesoría en la construcción de los instrumentos de evaluación que se usan al interior de los cursos (rúbricas), los cuales evalúan las competencias específicas. Adicional a la construcción de las rúbricas, el sistema continúa con el diseño y actualización de los instrumentos, y se encuentra en la planeación del año 2021. 
También se logró apoyar el reconocimiento de competencias previas de un grupo de personas que querían continuar sus estudios profesionales en el programa Crossmedia, provenientes de programas similares que ofrece el Servicio Nacional de Aprendizaje (SENA). La Universidad ha caracterizado los resultados de la población estudiantil, el desempeño de los estudiantes por campus, programa, región y competencias. Al 2019, con esta información (nueve momentos de prueba, doce instrumentos aplicados y 47.104 aplicaciones por parte de los estudiantes), se logró observar que la mayoría de los estudiantes se encuentra en un nivel multiestructural según la taxonomía SOLO usada por la Universidad. El objetivo principal del sistema se cumple, ya que provee a la comunidad información oportuna y confiable sobre los resultados del desempeño en las competencias que se desarrollan en la Universidad. Los estudiantes reciben en su correo institucional el informe con los resultados, máximo 5 días después del cierre de las pruebas, con información de su desempeño de manera puntual e histórica, con información numérica en escala de calificación, e información cualitativa de lo que implica el nivel obtenido en términos de la complejidad cognitiva de las acciones que demuestra. Además, el estudiante puede observar cómo y dónde se encuentra en relación con sus compañeros de programa, de campus y a nivel nacional.

Al final de cada informe el estudiante obtiene, según la descripción de los niveles previstos en la competencia, una hoja de ruta para que se trace la meta más próxima al nivel obtenido, y así haga de su experiencia de aprendizaje algo más real y alcanzable, de la mano de su profesor, compañeros, familia y en general de la institución. Esta misma información se encuentra en versión dirigida a las directivas académicas, profesores y administrativos en general.

\section{EVALUACIÓN Y REFLEXIÓN CRÍTICA}

La Universidad anualmente, en el comité de trabajo conformado por la dirección de Gestión de Programas, la jefatura del SIEC, la Vicerrectoría Académica y el asesor experto externo, analiza la experiencia obtenida y las características de sostenibilidad, pertinencia, y resultados arrojados por el SIEC. De manera interna, al término de cada aplicación, los instrumentos se evalúan bajo el procesamiento de Teoría de Respuesta al Ítem (TRI), procesamiento que permite verificar el correcto funcionamiento de instrumento y que arroja información vital para aplicar ajustes, retirar o transformar ítems y finalmente asignar escalas. En cuanto al Sistema, se han encontrado dificultades en torno a la custodia y almacenamiento 

está diseñando una manera de lograr transformar la dinámica para que la información sea susceptible de ser gestionada por las plataformas con las que se cuenta actualmente, a partir de desarrollos puntuales de software y en general arquitectura superior de datos, que permita que toda la información tenga un alto grado de seguridad y que, además, sea altamente transaccional. Como resultado de estas evaluaciones, se logró también reducir costos adelantando desarrollos con la plataforma LMS actual, para no incurrir en gastos adicionales de contratación de servicios tecnológicos para desplegar la aplicación de pruebas y la generación de reportes, situación que por dos años generó un importante costo para la universidad. Entre otros, las sesiones de análisis y evaluación han llevado a transformar la dinámica de los encuentros de las colegiaturas para el diseño de los instrumentos, actividad que se ha venido optimizando, a partir del uso cada vez mayor de la virtualidad en uso de sesiones sincrónicas y asincrónicas. Dos de las dificultades más notorias son: la sostenibilidad de los grupos de expertos en las colegiaturas, en términos de mantener una base mínima de profesionales que sean competentes en el diseño de los instrumentos de evaluación bajo las características que el sistema establece, ya que la movilidad de las personas laboralmente hablando, supone un reto para lograr establecer dinámicas muy bien estructuradas que se precien de ser replicables y sostenibles. Como segundo aspecto, es complejo cambiar en la comunidad educativa, especialmente estudiantes y profesores, la visión de la evaluación como un mero ejercicio de calificación, situación que lleva a los estudiantes a pedir una nota por cada momento evaluativo que se despliegue. Hemos logrado cambiar un poco esta visión, y seguimos construyendo una cultura de la evaluación como motor permanente de la discusión académica, en pro de mejorar el aprendizaje de los estudiantes, y por comprensión elevar la calidad de educación superior.

\subsection{DIVULGACIÓN}

El sistema llega a la comunidad a partir de una serie de medios según el público al que se dirige. Inicialmente toda la dinámica es observable por la comunidad interna y externa desde el micro-sitio del SIEC alojado en la página web de la universidad; en este espacio se encontrará información general del SIEC, así como accesos a las plataformas de pruebas, información de las áreas de trabajo y estructura en general. En cuanto a los resultados se divulgan de manera individual a cada una de las personas que aplican a las pruebas, ya sean estudiantes o profesores;

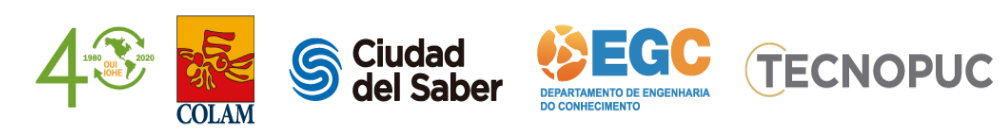


resultados de la prueba aplicada, así como el histórico de las pruebas anteriores y sus respectivos resultados. Además, se despliegan un reporte cualitativo sobre la descripción del nivel alcanzado y las escalas numéricas correspondientes. Lo anterior llega a cada una de las bandejas de correo institucional, bien sea para los profesores, estudiantes o administrativos. Para las directivas y grupos académicos, se envían reportes a manera de paneles de Power BI, en los que se presenta toda la información condensada estadísticamente y detallada según las necesidades de análisis. Esta información se discute en los diferentes consejos académicos de cada campus y los comités curriculares de cada programa. De la misma manera se entregan consolidados en forma de documento de Excel, para las personas que se encargan de trabajar datos de manera relacional y se generan consultas y diferentes reportes para los sistemas de información internos. La división de comunicaciones también despliega información desde los diferentes medios y redes y promueven el conocimiento de la información general, así como de la importancia que reviste la evaluación. 
ciki

X Congreso internacional de conocimiento e innovación

\section{AGRADECIMIENTOS}

Como participante en la construcción del sistema, coautor de la documentación del SIEC, líder actual del proceso, y finalmente como ponente de la experiencia, debo agradecer a la Universidad Cooperativa por creer en este proyecto, por apoyar incondicionalmente al sistema, y especialmente, por establecer un grandioso equipo que es el responsable de la existencia del SIEC; para:

El Dr. Daniel Bogoya, asesor experto externo y autor de la Arquitectura del Sistema; El Dr. Manuel Unigarro, Director de Gestión de Programas de la Universidad y líder permanente del SIEC;

La Mg. Beatriz Betancur, Subdirectora de Gestión de Programas y líder académica; El Mg. Milton Vásquez, líder académico y jefe del SIEC durante tres años;

El Mg. Carlos Restrepo, jefe del Observatorio del Conocimiento y líder del área de rúbricas;

Para todos ellos, y en general a todo el equipo de la Dirección de Gestión de Programas de la Universidad Cooperativa de Colombia, muchas gracias.

Johann Leonardo Campos Alvarado Jefe Sistema Institucional de Evaluación de Competencias Universidad Cooperativa de Colombia 2020 


\section{REFERENCIAS}

Bogoya, (2019). Sistema Institucional de Evaluación de Competencias, Arquitectura Conceptual. Universidad Cooperativa de Colombia.

Universidad Cooperativa de Colombia. Sistema Institucional de Evaluación de Competencias https://www.ucc.edu.co/academia/siec/Paginas/siec.aspx

Universidad Cooperativa de Colombia, Balance Social Informe de sostenibilidad.

https://www.ucc.edu.co/institucion/Paginas/balance-social.aspx 